\title{
Analysis of Tactical, Decisional and Executional Behaviour According to the Level of Expertise in Squash
}

\author{
by \\ María Catalán-Eslava1, Sixto González-Víllora², Juan C. Pastor-Vicedo, \\ Onofre R. Contreras-Jordán ${ }^{4}$
}

The objective of this study was to analyse the differences in tactical principles, decision-making and execution of the return shot and drop shot of squash players at different levels of proficiency. The sample was composed of 80 players, divided into four levels of expertise (first national category, second national category, autonomous regional level and provincial level). The Squash Performance Evaluation Tool was used for recording the level of decision-making and execution. The results revealed that reading of the context of the game improved as the level of the player's expertise of both the return shot and the drop shot increased. The correlation between correct decision-making and better quality execution for both the return shot and the drop shot in squash also improved with the player's level of expertise. According to these results, improvements in situational tactical principles and the application of tactical principles, correct decision-making and high-quality execution are essential to develop the athletic performance level among squash players.

Key words: tactical awareness, decision making, performance, evaluation, net games, sport.

\section{Introduction}

Technical, biomechanical and physiological factors are often referenced as a fundamental part of sports performance. However, cognitive processes that the athlete develops while practicing his/her sport are often not mentioned (García et al., 2009).

Sport performance is divided into cognitive components and those involving technique or skill. Cognitive components include knowledge and decision-making, whereas skill components correspond to motor execution (Iglesias Gallego et al., 2010). Therefore, the quality of decision-making in a game situation is as important as the execution of motor skills, and both are crucial to athletic performance (Blomqvist et al., 2001; González-Víllora et al., 2015a,b).

In this manner, high performance in the sport of squash depends on several factors, including tactics employed by the player, which is reflected in the wide variety of shots from which to choose, the execution of these technical skills in various parts of the court and in the specific circumstances required by the game at each particular moment (Vučković et al., 2004). These specific circumstances of the game are forever changing in squash, and thus, tactics and decision-making are very important in such open situations. Therefore, to achieve greater efficiency in decision-making, emphasis is placed on the importance of using taught strategies aimed at the recognition of contextual factors. This is accomplished by analysing tactical behaviour during games or using videos to enhance player's

\footnotetext{
1 - Ministry of Education, Culture and Sport. EDAF research group. Cuenca, Spain.

2 - Teacher Training Faculty, University of Castilla-la Mancha. EDAF research group. Cuenca, Spain.

3 - Teacher Training Faculty, University of Castilla-la Mancha. EDAF research group. Albacete, Spain.

${ }^{4}$ - Teacher Training Faculty, University of Castilla-la Mancha. EDAF research group. Albacete, Spain.
} 
tactical knowledge (McPherson, 2008).

Little research has been performed on squash as a sport; however, learning more about how players make decisions and how technicaltactical actions are correctly executed could improve future training in this sport. Understanding the decision-making function will help improve performance of experienced athletes and optimise training of novices (Vila-Maldonado et al., 2012).

Numerous areas of research study decision-making by athletes, and one of the most relevant approaches is decision-making according to cognitive psychology and ecological psychology (García et al., 2011). Both disciplines are scientifically based on the information processing theory; however, the cognitive paradigm seeks to understand the decision from the process that the player develops for solving tactical problems in his/her natural gaming environment (García et al., 2011), whereas the ecological paradigm studies the decisional behaviour from the subject's relationship with their sporting environment, based on continuous and active exploration and selection processes of the information relevant to the athlete's decision (Araújo et al., 2006).

The importance of using both of these paradigms is to understand and explain the decisional process to improve sports performance. In this way, cognitive perspective studies provide a number of resources that allow for better decisional capacity due to the influence of various variables, such as visual behaviour, temporal variables or prior knowledge of actions (Carvalho et al., 2011). Additionally, the ecological paradigm provides new approaches for the study of sport actions, such as the concept of a tactical action as a functional interaction between the individual and his/her sport, carried out with a specific purpose, situates the centre of scientific interest in the product of the sporting action and opens new perspectives to study the tactical behaviour of the athlete. Ecological studies apply the Theory of Direct Perception (Gibson, 1979), the Dynamical Systems Theory (Araújo, 2005), or the perceptionaction process (Fajen, 2007).

Therefore, despite their differences, both paradigms give much importance to decisionmaking, where the early beginnings of an athlete's conduct towards a particular task arise from adaptive processes, which allow the similarities and differences of both paradigms to be addressed.

For this reason, the present study sought to analyse the differences in tactical principles, decision-making and existing performance of the return shot and the drop shot in players of different levels of proficiency in squash. Players were grouped into four levels of expertise, and an analysis of a real-game situation was conducted to favour the nature of decision-making and its performance in the athlete's specific context (the squash court). The proposed hypothesis was that the players with higher levels of expertise would present better results (tactical principles, decisionmaking and execution) in a real game, both with the return shot as well as the drop shot, than players with a lower level of expertise.

\section{Methods}

\section{Participants}

The Ethics Committee from Albacete of the University of Castilla-la Mancha certify that the study was accomplished in accordance with the Spanish ethics regulatory laws, and did not need approval from the Ethics Review Committee. This research was an observational non-intervention study, with oral and written acceptance of the participants included, that were adult athletes. The study had no socio-affective aspect that morally limited its application. Before the data collection, the participants were informed of the research objectives and signed an informed consent form allowing data collection and their use for research purposes.

A total of 80 squash players (mean age $=$ $33.46 \pm 8.24$ years) volunteered to participate in the study. These subjects were grouped in four different categories according to the level of expertise, including level 'A', 'B', 'C' and 'D', each with $n=20$ players. These levels had been set by the Royal Spanish Squash Federation (Real Federación Española de Squash) to establish the senior national ranking as follows:

Level 'A': First category national players and those ranked in the senior national ranking from the first to twentieth position.

Level 'B': Second category national players and those ranked in the senior national ranking from the twenty-first position downwards. 
Level ' $\mathrm{C}$ ': Players competing at autonomous regional level; these players are federated but only compete regionally.

Level 'D': Non-federated players who compete at a provincial level; these are novice players in this sport.

The specific competitions where the analysis was performed for each participant group were:

Level 'A': Spanish National Squash Championship of absolute group; Alcobendas (Madrid, Spain); May, 2012.

Level 'B': Spanish National Squash Championship by Autonomy (by teams); Vigo (Spain); October, 2012.

Level 'C': 1) Castilla-la Mancha Regional Squash Championship; Cuenca (Spain); April, 2012. 2) Valencia Regional Squash Championship; Valencia (Spain); April, 2012.

Level 'D': 1) V San Julián Tournament; Cuenca (Spain); August, 2012. 2) CSC League; Cuenca (Spain); from October 2011 to May 2012.

\section{Measures}

The Squash Performance Evaluation Tool (HERS in Spanish) was used. This is an instrument for measuring individual performance in a real competition game of squash. It was adapted and validated (Catalán-Eslava et al., 2015) from the Tennis Game Components Coding Instrument (McPherson and French, 1991) and modified for Spain (Arias et al., 2011).

The evaluation tool distinguishes two fundamental moments of the game:

- The service, defined as the action that starts every game for match points (Rules of Squash game, Spanish Squash Federation, 2009).

- The game, defined as the interchange of hits to the ball after the serve until a point is achieved (Rules of Squash game, Spanish Squash Federation, 2009).

From these two stages, the service and the game, three aspects were evaluated: control, decision and execution. Furthermore, each of these three aspects was coded by the instrument, with values as follows:

- Control: from 0 (inappropriate control) to 1 (appropriate control).

- Decision: from 0 (inappropriate decision) to 1 (appropriate decision).

- Execution: from 0 (unforced error), 1 (forced error), 2 (a hit that does not force the opponent) to 3 (a point or hit that forces the opponent).

Evaluation of the sport of squash focuses on the moment of play. Within this stage, the technical-tactical hits of the serve return and the drop shot are analysed; the aspects evaluated in the game including the decision and the execution.

Analysing the service was decided against, as the serve is usually very restricted due to the limited space of the court (both the place of the server as well as the service box).

However, unlike the service, which is a closed gesture with little uncertainty and without a time limit to make and execute decisions, the analysis of the return and the drop shot was performed. The return shot is an open gesture when the player has all the regulatory space available to play the ball; the uncertainty generated for this shot is very high, and the reaction and execution time for any decision is very short. The drop shot was chosen because it represents an essential action for performance in squash, according to the current Squash Rules of the Game (2009). This shot changes the pace of the game, is physically wearing, and increases the pressure, anxiety and uncertainty of the opponent.

The control analysis tool evaluates the actions of play that have been encoded beforehand as having sufficient control, i.e., those actions of the game in which the player has a chance to decide and execute, ruling out any hits with the racket frame or irregular bounces of the ball. However, the use of the control analysis tool was dismissed during the moments of playing squash and control was not analysed in this study, as several investigations had shown no significant differences in control for players of different levels of expertise (McPherson, 1999; Nielsen and McPherson, 2001). Additionally, no significant differences were observed in the pilot studies prior to this research.

Finally, this study included a new aspect to be evaluated within the game: the contextualised role that the players acquire in this sport, such as the roles of an offensive or a defensive player with the technical-tactical actions of the return and the drop shot.

This new aspect brings racket sports closer to team sports, as it uses the same tactical 
principles of team sports (in attack (offense): keep the ball, progress towards the goal and achieve the objective; defensive (defence): retrieve the ball, stop the ball progressing forward, and prevent the goal) for both the game situation (the most appropriate action according to the context and situation at that moment of the game) and the application of skill (the action the player has decided to carry out and execute) (Bayer, 1992). When these principles are adapted to racket or net sports, they relate to keeping the ball in play, taking the initiative of the point and winning the point (Contreras-Jordán et al., 2007).

The tactical principles for the return and the drop shot were coded with values of one (keep the ball in play), two (take the lead of the point) and three (score the point).

\section{Procedures}

Eighty research subjects were recorded while playing a full competition game. A squash match consists of the best of three or five games (determined by the referee according to the type of competition). Every game is played to 11 points, and the player who scores 11 points first wins the game; however, when the score reaches 10-all, the game continues until one player leads (and so wins) by two points (Rules of Squash game, 2009).

To ensure the greatest possible accuracy in terms of recording the matches and obtaining the information, a protocol was established. This protocol consisted of placing the camera, stabilised on a tripod $(1 \mathrm{~m})$, at the highest point of the bleachers (spectator stands). The full game was recorded for each of the study participants. Recording did not stop during the rest periods, as they were $90 \mathrm{~s}$ long, and the camera zoom was not changed due to the size of the ball and the high speed of the competition. For the protocol used for data collection, a previously fixed number of 14 repeats of the technical-tactical elements of the return and the drop shot were used; this was performed in an attempt to balance the entire study sample, with a sufficient number of hits to be able to analyse the considered variables. All information was recorded on an observation sheet for each player, which contained all the hits and the sections according to categories (tactical principles, decision-making and execution).

\section{Statistical analysis}

The data recorded by the HERS were tested for normality, skewness and kurtosis. The results were evaluated using frequencies, histograms and the Kolmogorov-Smirnov $\mathrm{Z}$ test. As these tests presented significant differences between all of the studied variables, it was stated that the distribution was not normal. Therefore, the decision was made to perform nonparametric tests for the study.

The nonparametric tests used for the inferential analysis were the Mann-Whitney U test for possible differences between the different levels of expertise, but grouped into two levels (A and $C D ; A B$ and $C D ; A$ and $B C D$ ), and the Kruskall-Wallis test to identify possible differences between the levels of expertise, grouping the participants into three (A, BC and $\mathrm{C}$ ) or four levels (A, B, C and D). In addition, analysis of variance (ANOVA) was used on the significant variables. In all of the analyses, the asymptotic significance value was $p<0.05$.

\section{Results}

Descriptive analysis of the return and the drop shot

The results presented in Table 1 refer to the percentage distribution of the situation, application, decision-making and execution principles depending on the level of expertise, both for the return as well as the drop shot.

In relation to the return shot, according to the situation principle, the contextual situation of the serve return coincided with the expertise on all levels, characterised by the situational tactical principal of taking the lead of the point.

In the applicational principle, the players in skill levels A (experts) and B developed the technical-tactical action of the return mostly within the context of scoring the point $(44.15 \%$ and $35.32 \%$, respectively). In comparison, players in skill levels C and D (novices) developed the technical-tactical action of the return mainly in the context of taking the lead of the point $(38.67 \%$ and $42.46 \%$, respectively).

In regard to decision-making, the players in skill level A showed higher efficiency of correct decisions $(73.95 \%)$ compared to incorrect decisions (26.05\%). In comparison, players of skill levels B, C and D showed lower efficiency in correct decision-making compared to incorrect decision-making.

In executing the game, the players of all skill levels (A, B, C and D) presented a greater 
amount of shots that did not force the opponent.

The technical-tactical element of the drop shot, as shown in Table 1, revealed how the situational context of the drop shot was consistent at all levels of expertise, characterised by the situational tactical principal of taking the lead of the point.

For the applicational principle, the players of skill levels A and B developed the technical-tactical action of the drop shot, mainly in the context of taking the lead of the point (51.43\% and $49.45 \%$, respectively). In contrast, players of skill levels C and D mostly developed the technical-tactical action of the drop shot in the context of scoring the point $(52.04 \%$ and $45.24 \%$, respectively).

Regarding decision-making, all players, regardless of the skill level (A, B, C and D), were seen to take a higher percentage of correct decisions when it came to performing the technical-tactical action of the drop.

In executing the game, the players of all skill levels (A, B, C and D) presented a greater amount of shots that did not force the opponent.

Inferential analysis of the return and the drop shot

The results obtained from the inferential analysis of the return and the drop shot are presented in Table 2. To summarise these results, a significant amount of data are presented. First, these results present the variables that showed significant differences depending on the level of expertise (e.g., SP Keep, 001*). All nonsignificant variables were omitted; both sets of information are presented in the second column of Table 2. Second, within these variables the skill levels that produced intergroup differences were included for subsequent interpretation (e.g., SP Keep $\mathrm{D}<\mathrm{BC}$ ); this information is presented from the third to the seventh column in Table 2.

Thus, an inverse tendency was observed between the situational and the applicational context in squash, i.e., when significant differences were found in the situational context, no significant difference existed in the applicational context, and vice versa.

In decision-making, the players with a higher level of expertise (A and B) made fewer incorrect decisions compared to the players at skill levels $C$ and $D(A B<C D)$. In addition, the $D$ level players showed the highest number of unforced errors ( $D>A B C)$.

Regarding execution that did not force the opponent, it was found that higher skilled players in level A hit returns that did not force their opponent only on a few occasions $(A<B C D)$.

Level-A players (experts) hit the most returns that forced their opponent and that directly scored the point; level-B players (second national category) were the next most successful in these categories (Table 2). With respect to the drop shot, the results shown in Table 2 indicate that the tactical principle of taking the lead must be trained in a structured way for the technicaltactical gesture of the drop shot. The players with the highest level of expertise were clearly differentiated, as the analysis of the intergroup differences among other results showed $A B>C D$. Thus, the higher-level players (A and $\mathrm{B}$ ) were those who were tactically in a position to take the lead of the point on more occasions and were also the players who applied the tactical solution more often.

The results also showed that players from the highest level of expertise A (experts) and the players from level B (second national category) made the greatest number of incorrect decisions when taking a drop shot $(A B>C D)$.

In the execution of shots that did not force the opponent, the result of $\mathrm{AB}>\mathrm{CD}$ was obtained, which indicates that players of a higher level (A and B) performed more drop shots that did not force the opponent, compared to players of a reduced skill level (C and D).

In the execution of shots that forced the opponent or scored a direct point, the players with a higher skill level (A and B) performed drop shots that forced their opponent or that scored the point. In contrast, players of the lowest skill level $\mathrm{D}$ (novice) did not achieve this quality in the execution of the drop shot $(\mathrm{AB}>\mathrm{D})$.

Correlational analysis of the return shot and the drop shot

Tables 3 and 4 reflect the results of the correlation analysis of the return and the drop shot. In Table 3, the first and second rows of level A show the data related to taking the lead of the point (SP Take), with significant differences to the applicational principle of scoring the point, showing a significance value of $0.464^{*}$. This result shows that after reading a game situation where it was possible to take the lead of the point, level A 
players applied the principle of scoring the point. The other data are presented in the same manner, both for the tactical principles as well as between decision-making and the execution of the return and drop shots.

Tactical principles in the serve return action

In general, the results of the tactical principles correlation in the serve return showed that players with a higher skill level were better at reading the game and demonstrated superior technical-tactical application than the lower skilled players (Table 3). Players of higher skill levels (A and B) also showed positive correlations between the situational principle and the same tactical application principle or superior tactical principle $\left(0.464^{*} ; 0.704^{* *} ; 0.617^{* *} ; 0.345^{*}\right)$.
Decision-making and skill execution in the serve return

The results shown in Table 3 reveal that, for all players in the study sample, making the right decisions was positively correlated $\left(0.604^{* *} ; 0.471^{*} ; 0.550^{* *} ; 0.436^{* *} ; 0.367^{*} ; 0.491^{* *}\right)$ to the execution of shots that forced the opponent or scored the point directly. This result associates making correct decisions with higher quality executions, regardless of the level of the squash player's skill.

Table 1

Percentage distribution of the technical-tactical actions: the return and the drop shot

\begin{tabular}{lllll}
\hline Return & \multicolumn{4}{c}{ Players Skill Level } \\
\hline & $\mathrm{A}$ & $\mathrm{B}$ & $\mathrm{C}$ & $\mathrm{D}$ \\
\hline SP Keep & $14.70 \%$ & $20.40 \%$ & $15.80 \%$ & $2.00 \%$ \\
SP Take & $85.30 \%$ & $79.60 \%$ & $83.80 \%$ & $97.60 \%$ \\
SP Score & $0.00 \%$ & $0.00 \%$ & $0.40 \%$ & $0.40 \%$ \\
AP Keep & $18.87 \%$ & $30.28 \%$ & $28.00 \%$ & $30.18 \%$ \\
AP Take & $36.98 \%$ & $34.40 \%$ & $38.67 \%$ & $42.46 \%$ \\
AP Score & $44.15 \%$ & $35.32 \%$ & $33.33 \%$ & $27.37 \%$ \\
DM Incorrect & $26.05 \%$ & $51.02 \%$ & $60.15 \%$ & $69.05 \%$ \\
DM Correct & $73.95 \%$ & $48.98 \%$ & $39.85 \%$ & $30.95 \%$ \\
EXE Error & $2.52 \%$ & $0.51 \%$ & $5.64 \%$ & $14.29 \%$ \\
Forced EXE Error & $1.26 \%$ & $1.02 \%$ & $2.63 \%$ & $0.79 \%$ \\
Unforced EXE & $49.16 \%$ & $61.22 \%$ & $59.40 \%$ & $61.90 \%$ \\
Forced EXE & $47.06 \%$ & $37.24 \%$ & $32.33 \%$ & $23.02 \%$ \\
\hline Drop & & & & \\
\hline & & \multicolumn{2}{c}{ Players Skill Level } & \\
\hline SP Keep & $\mathrm{A}$ & $\mathrm{B}$ & $\mathrm{C}$ & $\mathrm{D}$ \\
SP Take & $3.93 \%$ & $6.59 \%$ & $6.12 \%$ & $17.86 \%$ \\
SP Score & $56.07 \%$ & $48.40 \%$ & $51.02 \%$ & $46.43 \%$ \\
AP Keep & $40.00 \%$ & $44.51 \%$ & $42.86 \%$ & $35.71 \%$ \\
AP Take & $5.36 \%$ & $5.49 \%$ & $11.22 \%$ & $19.05 \%$ \\
AP Score & $51.43 \%$ & $49.45 \%$ & $36.73 \%$ & $35.71 \%$ \\
DM Incorrect & $43.21 \%$ & $45.05 \%$ & $52.04 \%$ & $45.24 \%$ \\
DM Correct & $38.93 \%$ & $35.16 \%$ & $37.89 \%$ & $34.52 \%$ \\
Unforced EXE Error & $61.07 \%$ & $64.84 \%$ & $65.26 \%$ & $65.48 \%$ \\
Forced EXE Error & $8.57 \%$ & $9.89 \%$ & $13.78 \%$ & $11.90 \%$ \\
Unforced EXE & $1.43 \%$ & $2.75 \%$ & $3.57 \%$ & $7.14 \%$ \\
Forced EXE & $53.21 \%$ & $48.35 \%$ & $41.33 \%$ & $47.62 \%$ \\
\hline & $36.79 \%$ & $39.01 \%$ & $41.33 \%$ & $33.33 \%$ \\
\hline
\end{tabular}

$S P=$ Situational Principle; $A P=$ Applicational Principle;

$D M=$ Decision-Making; EXE = Execution 
Table 2

Inferential analysis of the technical-tactical actions in squash

\begin{tabular}{|c|c|c|c|c|c|c|c|}
\hline Return & A-B-C-D & \multicolumn{2}{|l|}{ A-BC-D } & AB-C-D & A-BCD & $\mathrm{AB}-\mathrm{CD}$ & $\mathrm{A}-\mathrm{CD}$ \\
\hline \multirow[b]{2}{*}{ SP Keep } & $.001^{*}$ & \multicolumn{2}{|l|}{$.000^{*}$} & $.000^{*}$ & .452 & $.004^{*}$ & .083 \\
\hline & $\mathrm{D}<\mathrm{B} . \mathrm{C}$ & \multicolumn{2}{|l|}{$\mathrm{BC}>\mathrm{A}>\mathrm{D}$} & D<AB.C & & $\mathrm{AB}>\mathrm{CD}$ & \\
\hline \multirow[b]{2}{*}{ SP Take } & $.001^{*}$ & \multicolumn{2}{|l|}{$.001^{*}$} & $.000^{*}$ & .250 & $.001^{*}$ & $.021^{*}$ \\
\hline & $\mathrm{D}>\mathrm{B}$ & \multicolumn{2}{|l|}{$\mathrm{D}>\mathrm{A} . \mathrm{BC}$} & $\mathrm{D}>\mathrm{AB}$ & & $\mathrm{AB}<\mathrm{CD}$ & $\mathrm{A}<\mathrm{CD}$ \\
\hline \multirow[b]{2}{*}{ AP Keep } & .070 & \multirow{2}{*}{\multicolumn{2}{|c|}{$\begin{array}{l}.037^{*} \\
\mathrm{D}>\mathrm{A}\end{array}$}} & .087 & $.013^{*}$ & $.028^{*}$ & $.007^{*}$ \\
\hline & & & & & $\mathrm{A}<\mathrm{BCD}$ & $\mathrm{AB}<\mathrm{CD}$ & $\mathrm{A}<\mathrm{CD}$ \\
\hline \multirow[b]{2}{*}{ AP Score } & $.003^{*}$ & \multicolumn{2}{|l|}{$.001^{*}$} & $.003^{*}$ & $.003^{*}$ & $.003^{*}$ & $.001^{*}$ \\
\hline & $\mathrm{A}>\mathrm{D}$ & \multicolumn{2}{|l|}{$\mathrm{A}>\mathrm{BC} . \mathrm{D}$} & $A B>D$ & $\mathrm{~A}>\mathrm{BCD}$ & $\mathrm{AB}>\mathrm{CD}$ & $\mathrm{A}>\mathrm{CD}$ \\
\hline \multirow{3}{*}{ DM Incorrect } & $.000^{*}$ & \multirow{3}{*}{\multicolumn{2}{|c|}{$\begin{array}{l}.000^{*} \\
\mathrm{~A}<\mathrm{BC} . \mathrm{D} \\
\mathrm{D}>\mathrm{A} . \mathrm{BC}\end{array}$}} & $.000^{*}$ & $.000^{*}$ & $.000^{*}$ & $.000^{*}$ \\
\hline & A<B.C.D & & & $A B<C . D$ & $\mathrm{~A}<\mathrm{BCD}$ & $\mathrm{AB}<\mathrm{CD}$ & $\mathrm{A}<\mathrm{CD}$ \\
\hline & & & & & & & \\
\hline \multirow[b]{2}{*}{ DM Correct } & $.000^{*}$ & \multicolumn{2}{|l|}{$.000^{*}$} & $.001^{*}$ & $.000^{*}$ & $.001^{*}$ & $.000^{*}$ \\
\hline & A>B.C.D & \multicolumn{2}{|l|}{$\mathrm{A}>\mathrm{BC} . \mathrm{D}$} & $A B>D$ & $\mathrm{~A}>\mathrm{BCD}$ & $\mathrm{AB}>\mathrm{CD}$ & $\mathrm{A}>\mathrm{CD}$ \\
\hline \multirow[b]{2}{*}{ Unforced Error } & $.000^{*}$ & \multirow{2}{*}{\multicolumn{2}{|c|}{$\begin{array}{l}.000^{*} \\
D>A . B C\end{array}$}} & $.000^{*}$ & .069 & $.000^{*}$ & $.003^{*}$ \\
\hline & D>A.B.C & & & D>AB.C & & $\mathrm{AB}<\mathrm{CD}$ & $\mathrm{A}<\mathrm{CD}$ \\
\hline \multirow{2}{*}{\multicolumn{2}{|c|}{ Unforced Execution }} & \multirow{2}{*}{\multicolumn{2}{|c|}{$\begin{array}{l}.033^{*} \\
\mathrm{~A}<\mathrm{BC} . \mathrm{D}\end{array}$}} & .067 & $.011^{*}$ & $.020^{*}$ & $.006^{*}$ \\
\hline & & & & & $\mathrm{A}<\mathrm{BCD}$ & $\mathrm{AB}<\mathrm{CD}$ & $\mathrm{A}<\mathrm{CD}$ \\
\hline \multirow{3}{*}{ Forced Execution } & \multirow{3}{*}{$\begin{array}{l}.002^{*} \\
\mathrm{~A}>\mathrm{D}\end{array}$} & \multirow{3}{*}{\multicolumn{2}{|c|}{$\begin{array}{l}.001^{*} \\
\mathrm{~A}>\mathrm{BC} . \mathrm{D} \\
\mathrm{D}<\mathrm{A} . \mathrm{BC}\end{array}$}} & $.001^{*}$ & $.003^{*}$ & $.002^{*}$ & $.001^{*}$ \\
\hline & & & & $A B>D$ & $\mathrm{~A}>\mathrm{BCD}$ & $\mathrm{AB}>\mathrm{CD}$ & $\mathrm{A}>\mathrm{CD}$ \\
\hline & & & & & & & \\
\hline \multirow{3}{*}{$\frac{\text { Drop }}{\text { SP Take }}$} & A-B-C-D & A-BC-D & AB-C-D & & A-BCD & $\mathrm{AB}-\mathrm{CD}$ & $\mathrm{A}-\mathrm{CD}$ \\
\hline & $.001^{*}$ & $.000^{*}$ & $.003^{*}$ & & $.001^{*}$ & $.009^{*}$ & $.001^{*}$ \\
\hline & $A>D$ & $\mathrm{D}<\mathrm{A} . \mathrm{BC}$ & $\mathrm{AB}>\mathrm{D}$ & & $\mathrm{A}>\mathrm{BCD}$ & $\mathrm{AB}>\mathrm{CD}$ & $A>C D$ \\
\hline \multirow[t]{2}{*}{ AP Take } & $.000^{*}$ & $.000^{*}$ & $.000^{*}$ & & $.000^{*}$ & $.000^{*}$ & $.000^{*}$ \\
\hline & $\mathrm{A}>\mathrm{C} . \mathrm{D} B>\mathrm{D}$ & $\mathrm{A}>\mathrm{BC} . \mathrm{D}$ & $\mathrm{AB}>\mathrm{C} . \mathrm{D}$ & & $\mathrm{A}>\mathrm{BCD}$ & $\mathrm{AB}>\mathrm{CD}$ & $\mathrm{A}>\mathrm{CD}$ \\
\hline \multirow[t]{2}{*}{ DM Incorrect } & $.007^{*}$ & $.003^{*}$ & $.010^{*}$ & & $.009^{*}$ & $.035^{*}$ & $.008^{*}$ \\
\hline & $\mathrm{A}>\mathrm{D}$ & $\mathrm{A}>\mathrm{D}$ & $\mathrm{AB}>\mathrm{D}$ & & $\mathrm{A}>\mathrm{BCD}$ & $\mathrm{AB}>\mathrm{CD}$ & $A>C D$ \\
\hline \multirow[t]{2}{*}{ Unforced Execution } & $.004^{*}$ & $.001^{*}$ & $.009^{*}$ & & $.001^{*}$ & $.005^{*}$ & $.001^{*}$ \\
\hline & $A>D$ & $\mathrm{~A}>\mathrm{BC} . \mathrm{D}$ & $\mathrm{AB}>\mathrm{D}$ & & $\mathrm{A}>\mathrm{BCD}$ & $\mathrm{AB}>\mathrm{CD}$ & $A>C D$ \\
\hline \multirow[t]{2}{*}{ Forced Execution } & $.021^{*}$ & $.008^{*}$ & $.008^{*}$ & & .224 & $.041^{*}$ & .102 \\
\hline & $A>D$ & $\mathrm{D}<\mathrm{A} . \mathrm{BC}$ & $\mathrm{AB}>\mathrm{D}$ & & & $\mathrm{AB}>\mathrm{CD}$ & \\
\hline
\end{tabular}

$S P=$ Situational Principle $; A P=$ Applicational Principle;

$D M=$ Decision-Making; EXE = Execution 
Table 3

Correlational analysis of the technical-tactical action of the return in squash

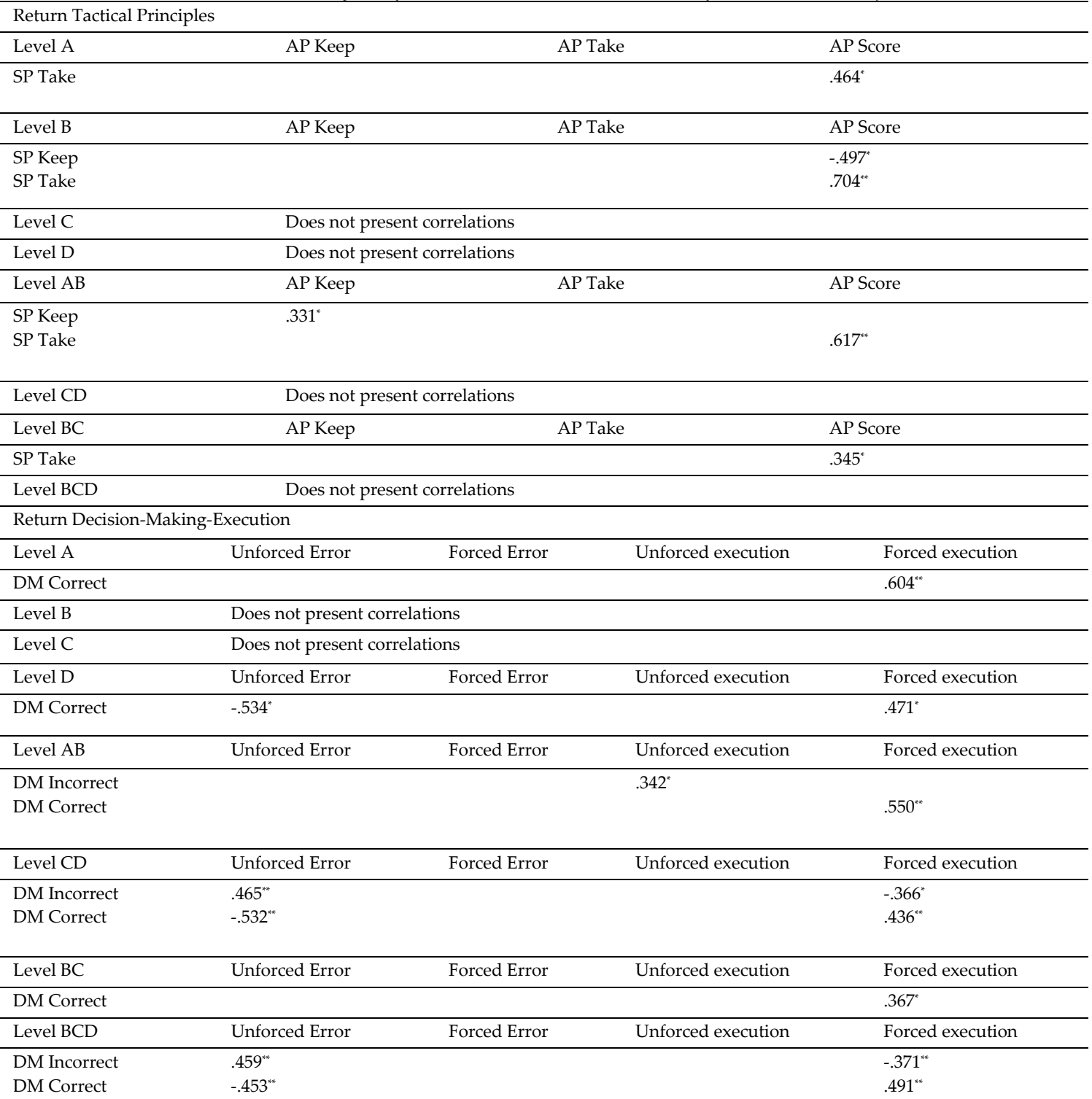

\footnotetext{
$S P=$ Situational Principle $A P=$ Applicational Principle $; D M=$ Decision-Making; $E X E=$ Execution
} 
Table 4

Correlational analysis of the technical-tactical action of the drop in squash

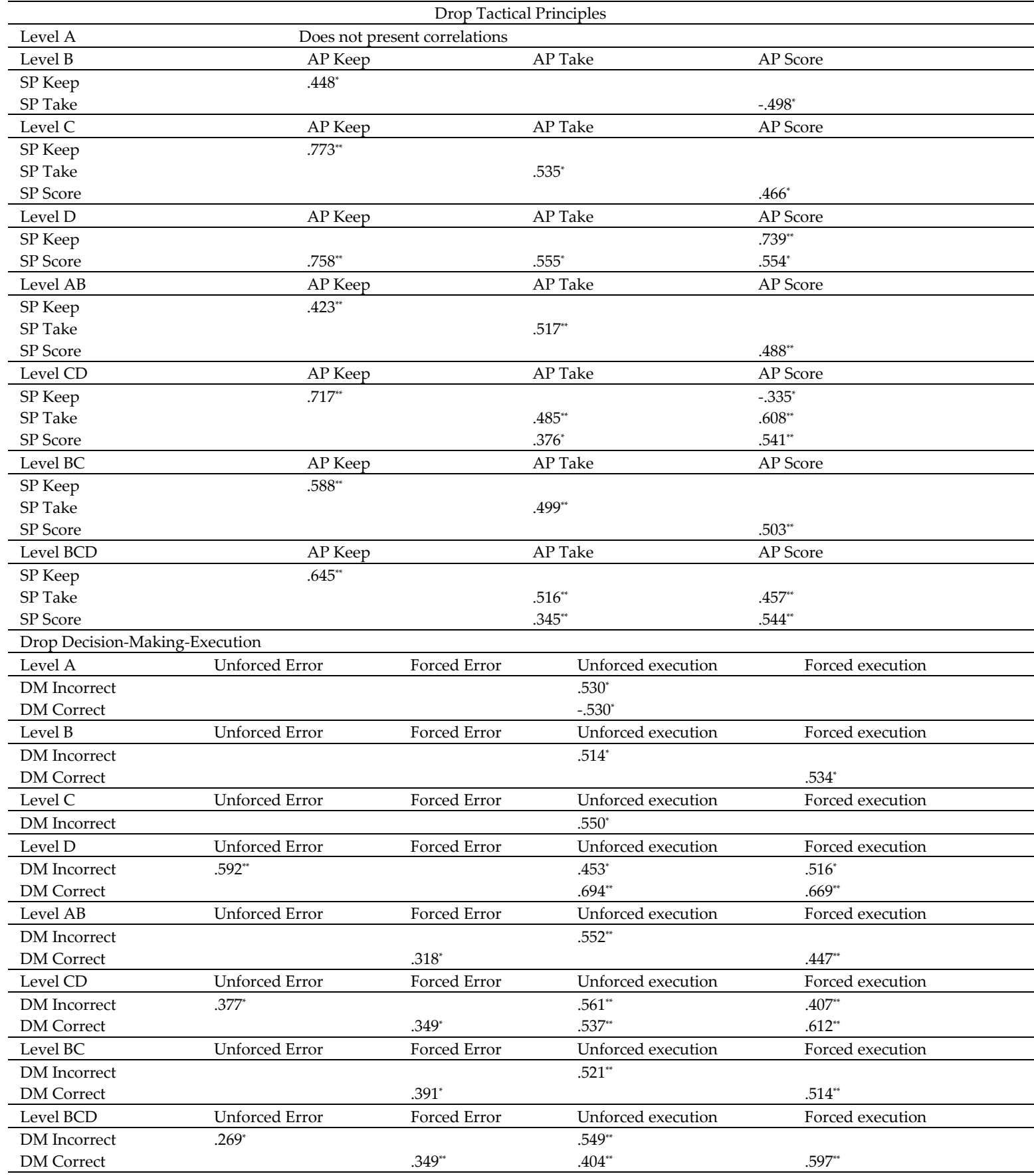

$S P=$ Situational Principle $; A P=$ Applicational Principle $; D M=$ Decision-Making $;$ EXE = Execution 
Tactical principles of the drop shot

The results regarding the tactical principles of the drop shot were extremely varied (Table 4), which suggests that the highest level of expertise (level A) is not correlated with tactical principles. However, there were significant positive correlations between all the situational and applicational principles of second category national players (level B) and regional level players (level C).

Decision-making and execution of the drop shot

In summary, as shown in Table 4, the results of the drop shot correlations for decisionmaking and execution were varied for all players in the study sample.

\section{Discussion}

The objective of the current study was to analyse differences in the tactical principles, decision-making and execution of the return shot and the drop shot between squash players of different levels of expertise, thereby continuing the lines of scientific research conducted previously in other sports (González-Víllora et al., 2011, 2015a,b; Gutiérrez et al., 2011). The research presented in this paper evaluated the tactical behaviour in squash players to provide scientific knowledge about a sport that has not been often studied.

The obtained results reflect how expert players stand out above the rest of the skill levels, from the tactical application of taking the lead of the point or scoring the point, regardless of the previous tactical situation. In contrast, novice players apply the least number of offensive and complex tactical principles of action.

These results are similar to those of a previously published study (Vučković et al., 2009) on the use of the central area of the squash court (T), where the results showed that players who won a game dominated a larger part of the total playing time in the $\mathrm{T}$ area, compared to those players who lost. Similarly, we developed the study of the relationship between the technicaltactical actions applied and their dependence on the location of the player on the court and the elapsed time between hits (Vučković et al., 2013).

These noticeable differences in the comparison between expert and novice players were similar to those found in tennis players. Tennis, like squash, is a racquet sport, where expert players develop more varied knowledge than novice players, and the concepts of technicaltactical actions are developed with a greater number of aspects, which involve more sophisticated tactical knowledge by expert players (Arias et al., 2011).

Similarly, compared to novices, expert tennis players have superior tactical knowledge and are more flexible to the situation they find themselves in (McPherson and Kernodle, 2003). This aspect was also confirmed in our study, as squash players with lower skill levels (C and D, regional and novice level, respectively) presented applicational tactical principles that were less suited to the game situation compared to squash players with a higher level of expertise (A and B, experts and second national category, respectively).

Regarding decision-making based on the squash player's level of expertise in the technical-tactical gesture to the serve return, the results showed that experts made the highest number of correct decisions and the lowest number of incorrect decisions. Meanwhile, novice players made the highest number of incorrect decisions and the lowest number of correct decisions. This result is in agreement with a study of 12 tennis players (6 experts and 6 novices) showing differences between experts and novices (García et al., 2009). Applying an adaptation of the Tennis Game Components Instrument (McPherson and French, 1991), it was stated that the expert tennis players presented significantly better scores than novices in all of the decision variables of the technical-tactical gesture of the service and the rest of the strokes.

The second part of the analysis regarding decision-making based on the level of expertise in squash players also included the technical-tactical gesture of the drop shot. These results showed that the expert squash players performed a larger number of incorrect decisions and the lowest number of correct decisions, while the novice players made a larger number of correct decisions and less incorrect decisions. These results confirm the difference in the skill level between experts and novices. As the level of expertise increases, players execute more sophisticated decision-making, using the technical-tactical gesture for the drop shot with various purposes such as to change the 
opponent's pace, to fatigue the opponent, to reduce the opponent's anticipation, or to hinder the opponent's tactical positioning.

These strategies allow the player to prepare the point or to take the lead in the game to achieve the point in the medium- or long-term. Similarly, tennis players with the highest level of expertise consider more effective play possibilities, as they place greater force on their opponent (tactical responses) due to considering plays such as moving the opponent from one side of the court to the other, playing the weak side, or playing short balls to make the drop shots more risky, as occurs with expert squash players (Nielsen and McPherson, 2001). However, novice players make a greater number of correct decisions and a lower number of incorrect decisions because they do not have this technicaltactical security due to their lower level of expertise, i.e., their game dynamics on a decisional-cognitive level are poorer using the drop shot purely with the aim of gaining a point.

The third segment of our study investigated the differences in the execution of technical-tactical skills of the return and the drop shot depending on the player's level of expertise. In the technical-tactical gesture of the serve return, the expert squash players forced their opponent and achieved a direct point on more occasions than the novice players. In addition, in the technical-tactical gesture of the drop shot, the experts committed fewer mistakes and executions that did not force their opponent, compared to novice players. Similar differences in the performance level between expert and novice squash players in the execution of skills during a real game were also observed in a study with tennis players (García et al., 2009), where the expert players developed more successful shots both in the serve as well as return shots, and this result was consistent with the athletic performance shown by the players.

Results in other studies also reflect these differences between expert and novice players, where expert tennis players develop a greater number of suitable shots than novice players. This higher level can be explained by the fact that the expert tennis players use specific sport strategies that allow them to perform better (McPherson, 2008; Nielsen and McPherson, 2001). Additionally, in the two types of technical-tactical gestures observed, the expert players made decisions that were appropriate to a given situation, meaning that the skilled players not only performed better hits but also made tactically superior selections. This finding implies that experts can select the best shot and choose the most appropriate direction, and these data are similar to those obtained in other studies on similar characteristics with tennis players (García et al., 2009; Nielsen and McPherson, 2001).

Finally, to delve further into the study of decisional-cognitive behaviour in the sport of squash, a research hypothesis was proposed that the players with a higher level of expertise would present with better performance in the three fundamental areas of tactical principles, decisionmaking and execution in a real game situation, both in the return and the drop shot, compared to players with a lower level of expertise.

This hypothesis was confirmed, as the relationship between the situational principles and the applicable principles in the return and the drop shot indicated that higher skilled players had a more favourable relationship between their tactical principles at the moment of reading the game, which was absent in players at the lower expertise level.

The results obtained regarding the game strategy of higher-level players in squash coincide with those of other studies (Dodds et al., 2001), which have reported that expert players select and apply their knowledge of situations in which they find themselves in a more suitable manner and that they identify, remember or manage the relevant information more accurately to achieve the objective of the game (García et al., 2007).

The hypothesis regarding correlations between decision-making and execution of skills in both technical-tactical gestures (return and drop) was also confirmed. A narrow and optimum relationship between making correct decisions and the best performance, both in the return and in the drop, could be observed. Conversely, when the decision was incorrect, the execution of skills worsened.

Similar to the results for tactical principles, players of a higher skill level (experts and second national category) showed an adequate relationship between decisions and execution, unlike players at a lower skill level 
(regional and novice levels). The relationship between decisional-cognitive performance and the skill level of execution by players with a higher level of expertise may result from the experts not only knowing what to do in a variety of situations, but also knowing how and when to apply their theoretical and procedural knowledge and reproduce it in appropriate actions (Araújo and Serpa, 1998; González-Víllora et al., 2015; Gutiérrez et al., 2011). Furthermore, experts differ from novices in the amount and type of knowledge they possess as well as how this information is used when making decisions (Davids et al., 1999; Del Villar et al., 2014).

\section{Conclusions}

Our analysis of tactical, decisional and executional behaviour in the technical-tactical gesture of the serve return in a real game situation revealed the following conclusions:

- there existed an inverse tendency between situational tactical principles and applicational tactical principles;

- there existed a positive tendency between the level of expertise and correct decision-making, in such a way that as the level of expertise rose, the player's decisional capacity was more appropriate;

- execution of the return distinguished between squash players of different performance levels of; to be able to move up a level in expertise in squash, every return shot must force the opponent.

In addition, our analysis of the tactical, decisional and executional behaviour in the technical-tactical gesture of the drop shot in a real game situation revealed the following conclusions:

- the tactical principle of taking the lead of the point with the drop shot differentiated between different levels of expertise among squash players;
- making correct decisions did not show significant differences between skill groups and was therefore not a component that distinguished between the squash player's level of expertise. However, significant differences in making incorrect decisions were found, with a positive tendency of making incorrect decisions at a higher level of expertise;

- execution of the drop shot was a characteristic feature needed to reach the expert level of expertise, i.e., the drop was shown to be a technical-tactical action that marked the difference of the skill level in squash.

Finally, our hypothesis was confirmed for the three variables and both technical-tactical gestures studied. In particular, the correlation between the situational tactical principles and the applicational tactical principles revealed that the greater the player's level of expertise in both the return as well as the drop shot, the better the reading of the game context. The correlation between correct decision-making and betterquality execution was confirmed scientifically for both the return and the drop shot.

It is important to conduct this type of research in sports that are minority modalities and are not often studied rather than just focusing on dominant sports and those where the aspects regarding scientific knowledge about cognitive, decision-making and execution of the skills are already known. Analysis of net and wall modalities, such as in squash, opens the door for new research to expand the Squash Performance Evaluation Tool, which is valid and reliable for analysing technical-tactical gestures of the serve return and the drop shot in real-game situations, to develop a comprehensive tool and a computerised system for assessing the technical and tactical performance in real-game situations. However, it would also be of interest to perform this type of study with a female study sample.

\section{References}

Araújo D. The tactical action in sport: an overview in Rosado A, Araújo D, Colaço C. The decision context. Lisboa: Visão e Contextos, 21-33; 2005

Araújo D, Davids K, Hristovski R. The ecological dynamics of decision making in sport. Psychol Sport Exerc, 2006; 7: 653-676 
Araújo D, Serpa S. Dynamic decision making at different levels of expertise in the sport of sailing. Rev Psicología Deporte, 1998; 8: 103-115

Arias AG, Arroyo MPM, Domínguez AM, García L, Del Villar F. The federated practice as element of development of the knowledge: application to the formation volleyball. Rev Int Cienc Deporte, 2011; 7 : 230-245

Bayer C. The teaching of collective sports games. Barcelona: Hispano Europea; 1992

Blomqvist M, Luhtanen P, Laakso L. Comparison of two types of instruction in badminton. Phys Educ Sport Pedagogy, 2001; 6: 139-155

Carvalho J, Araújo D, García-González L, Iglesias D. Decision-making training in tennis: what scientific principles can be applied to training programmes? Rev Psicología Deporte, 2011; 20: 767-783

Catalán-Eslava M, González-Víllora S. Validation of a wall-net sports measurement instrument: squash performance evaluation tool (SPET). Retos, 2015; 27: 73-80

Contreras-Jordán OR, García-López LM, Gutiérrez D, Del Valle S, Rubio RMA. Introduction to racquet sports. Barcelona: Paidotribo; 2007

Davids K, Williams AM, Williams JG. Visual perception and action in sport. London: E \& FN Spon; 1999

Del Villar F, Iglesias D, Moreno MP, Fuentes JP, Cervelló E. An investigation into procedural knowledge and decision-making: Spanish experienced-inexperienced basketball players differences. J Human Mov Stud, 2014; 46: 407-420

Dodds P, Griffin LL, Placek JH. A selected review of the literature on development of learners' domainspecific knowledge. J Teach Phys Educ, 2001; 20: 301-313

Fajen BR. Affordance-based control of visually guided action. Ecol Psychol, 2007; 19: 383-410

García L, Araújo D, Carvalho J, Iglesias D. An overview of theories and research methods on decision making in tennis. Rev Psicología Deporte, 2011; 20: 645-666

García L, Iglesias D, Moreno MP, Moreno A, Del Villar F. Cognitive strategies developed by tennis players of different expertise level during game play. Apunts, 2007; 89: 40-47

García LG, Arroyo MPM, Domínguez AM, Gallego DI, del Villar Álvarez F. Relation between knowledge and decision making in tennis players and its influence in sport expertise. Int J Sport Sci, 2009; 17: 6075

Gibson JJ. The ecological approach to visual perception. Boston: Houghton Mifflin; 1979

González-Víllora S, García-López LM, Pastor-Vicedo JC, Contreras-Jordán OR. Tactical knowledge and decision making in young football players (10 years old). Rev Psicología Deporte, 2011; 20: 79-97

González-Víllora S, García-López LM, Contreras-Jordán OR. Decision making and skill development in youth football players. Int J Med Sci Phy Act Sport, 2015a; 15(59): 467-487

González-Víllora S, Serra-Olivares J, Pastor-Vicedo JC, Teoldo, I. Review of the tactical evaluation tools for youth players, assessing the tactics in team sports: football. SpringerPlus, 2015b; 4: 663.

Gutiérrez DG, González-Víllora S, García-López LM, Mitchell S. Differences in decision-making development between expert and novice invasion game players. Percept Mot Skills, 2011; 112: 871-888

Iglesias Gallego D, García González L, García Calvo T, León del Barco B, Villar Álvarez FD. Expertise development in sport: contributions under cognitive psychology perspective. J Hum Sport $\mathcal{E}$ Ex, 2010; 5(3): 462-475

McPherson SL. Expert-novice differences in performance skills and problem representations of youth and adults during tennis competition. Res Q Exerc Sport, 1999; 70: 233-251 McPherson SL. Tactics: using knowledge to enhance performance in Farrow D, Baker J, MacMahon C. Developing sport expertise: researchers and coaches put theory into practice. London: Routledge, 155-167; 2008

McPherson SL, French KE. Changes in cognitive strategies and motor skill in tennis. J Sport and Ex Psychol, 1991; 13: 26-41

McPherson SL, Kernodle MW. Tactics, the neglected attribute of expertise: problem representations and performance skills in tennis in Starkes JL, Ericsson KA. Expert performance in sports: advances in research on sport expertise. Champaign: Human Kinetics, 137-168; 2003

Nielsen TM, McPherson SL. Response selection and execution skills of professionals and novices during singles tennis competition. Percept Mot Skills, 2001; 93: 541-555 
Rules of Squash game. Real Federación Española de Squash. Recent Findings, 2009. Available at: http://www.rfesquash.es/; accessed on 01.04.2015

Vila-Maldonado S, García-López G, Contrerás-Jordán OR The research of the visula behabiour, form the cognitive-perceptual focus and the decision making in sports. J Sport Health Res, 2012; 2: 137-156

Vučković G, Dezman B, Erčulj F, Kovačič S, Perš J. Differences between the winning and the losing players in a squash game in terms of distance covered in Lees A, Kahn J-F, Maynard IW. Science and Racket Sports III. London: Routledge, 202-207; 2004

Vučković G, James N, Hughes M, Murray S, Sporiš G, Perš J. The effect of court location and available time on the tactical shot selection of elite squash players. J Sports Sci Med, 2013; 12: 66-73

Vučković G, Perš J, James N, Hughes M. Tactical use of the T area in squash by players of differing standard. J Sports Sci, 2009; 27: 863-871

\section{Corresponding author:}

\section{Sixto González-Víllora}

Teacher Training Faculty of Cuenca. University of Castilla-la Mancha

Edificio Fray Luís de León, Campus Universitario, s/n, 16071, Cuenca, Spain

Phone: +34 969179170 (4725)

Fax: +34902204130

E-mail: sixto.gonzalez@uclm.es 